\title{
Theoretical Investigation on Structural and Electronic Properties of InN Growth on Ce-Stabilized Zirconia (111) Substrates
}

\author{
Yao Guo, ${ }^{1}$ Taixuan Jia, ${ }^{1}$ Chengbo Li, ${ }^{2}$ Yongsheng Niu, ${ }^{1}$ \\ Shaogang Hou, ${ }^{1}$ and Shuanjiang Liu ${ }^{2}$ \\ ${ }^{1}$ Department of Chemistry and Environment Engineering, Anyang Institute of Technology, Anyang 455000, China \\ ${ }^{2}$ Department of Mathematics and Physics, Anyang Institute of Technology, Anyang 455000, China
}

Correspondence should be addressed to Yao Guo; guoyao@ayit.edu.cn

Received 21 November 2015; Accepted 23 February 2016

Academic Editor: Da-Ren Hang

Copyright (C) 2016 Yao Guo et al. This is an open access article distributed under the Creative Commons Attribution License, which permits unrestricted use, distribution, and reproduction in any medium, provided the original work is properly cited.

The structural and electronic properties of InN on Ce-stabilized zirconia (CeSZ) (111) substrates are investigated using firstprinciples calculations based on density functional theory with GGA $+U$ method. Surface energy calculations indicate that the structure of Ce-segregated surface is more energetically stable than that of Ce-segregation-free surface. Adsorption energies of indium and nitrogen atoms on both Ce-segregated and Ce-segregation-free CeSZ (111) surfaces at the initial growth stage have been studied. The results suggest that the first layer of InN films consists of a nitrogen layer, which leads to epitaxial relationships between InN (0001) // CeSZ (111) and InN [1120] // CeSZ [1ํㅣ‥ In addition, density of states (DOS) analysis revealed that the hybridization effect plays a crucial role in determining the interface structure for the growth of InN on CeSZ (111) surfaces. Furthermore, adsorption energies of indium atoms on the nitrogen layer have also been evaluated in order to investigate the lattice polarity determination for InN films. It was found that an indium atom preferentially adsorbs at the center of three nitrogen atoms stacked on the CeSZ substrate, which results in the formation of In-polarity InN.

\section{Introduction}

Indium nitride $(\mathrm{InN})$ is a promising material for high-speed electronic devices owing to its highest electron mobility [1-3] and saturation drift velocity [4] among the group III nitride. Growth of high-quality ultrathin InN films is necessary for the fabrication of InN-based devices since the device performance is often restricted by the threading dislocations and stacking faults at the interface [5]. Foreign substrates such as sapphire or GaN $[6,7]$ are usually employed for epitaxial growth of InN because of the lack of bulk InN crystals. However, the large lattice mismatches between InN and these substrates cause the formation of a high density of threading dislocations. In order to solve the problem above, development of lattice-matched substrates for $\mathrm{InN}$ is required. Among the various substrates, cubic phase zirconia substrates serve as a promising candidate for $\mathrm{InN}$ growth because they share a threefold rotational symmetry with the $\operatorname{InN}(0001)$ surface and have small lattice mismatch [8]. For example, the use of yttria-stabilized zirconia (YSZ) substrates provides reduced defect density and abrupt InN/YSZ interfaces [9]. In fact, the fluorite-type zirconia could be stabilized by the addition of different dopants. The stabilized cubic zirconia has its own characteristic structures which are dopant-independent [10]. The introduction of new dopants into zirconia that replaces yttrium is a straightforward strategy because the interaction between the dopant and the InN film is quite different.

Ceria-stabilized zirconia (CeSZ) is known as heterogeneous catalysis in the area of automotive pollution control [11]. On the other side, CeSZ is an ideal substrate owing to the small lattice mismatch between the InN film and the substrate. In fact, it has been experimentally determined that Ce atoms stabilize the cubic structure of zirconia and 
the Ce dopant segregation on the CeSZ (111) surface could be obtained due to the cation redistribution at high temperatures [12]. However, the feasibility of epitaxial growth of InN films on CeSZ (111) has not yet been investigated to date. Hence, a clear understanding of the InN/CeSZ interface microstructure is especially meaningful. To investigate the influence of the interfacial structure on electronic properties and the possible epitaxial relationships in the interface, the mechanism of growth of InN layers on CeSZ (111) substrates is discussed on the basis of first-principles calculations.

\section{Computational Method}

The theoretical calculations have been carried out in the framework of the ab initio density functional theory using the software MedeA VASP package [13]. The electron exchange and correlation were treated within the generalized gradient approximation (GGA-PBE) [14]. The wavefunctions were expanded in a plane-wave basis set with a cutoff energy of $800 \mathrm{eV}$. The electron-ion interactions were described by the full-potential PAW method [15]. The electron configurations of the pseudopotentials for In, N, Zr, Ce, and $\mathrm{O}$ are $4 d^{10} 5 s^{2} 5 p^{1}, 2 s^{2} 2 p^{3}, 4 s^{2} 4 p^{6} 4 d^{2} 5 s^{2}, 5 s^{2} 5 p^{6} 4 f^{1} 5 d^{1} 6 s^{2}$, and $2 s^{2} 2 p^{4}$, respectively. All calculations involving Ce were performed at the GGA $+U$ level with a Hubbard parameter $U_{\text {eff }}=5 \mathrm{eV}$ for the Ce $f$ orbitals [16]. The oxygen-terminated surface was employed because it was more stable than the $\mathrm{Zr}$ terminated or O-O terminated [17]. The slab contained twelve atomic layers (four O-Zr-O trilayers) separated from each other by a vacuum layer of $15 \AA$ in the perpendicular direction to eliminate any interactions between the adjacent slabs. In the optimization process, the layers in the bottom $\mathrm{O}-\mathrm{Zr}-\mathrm{O}$ trilayer were fixed at the bulk positions, while the top layers were fully relaxed with convergence criteria of $0.05 \mathrm{eV} / \AA$. The supercell consisted of a $2 \times 2$ array of CeSZ (111) surface unit cells. The Brillouin-zone integration was calculated with a $2 \times$ $2 \times 1 \mathrm{k}$-point grid, which was generated using the MonkhorstPack method [18]. We also performed the test with a denser $4 \times 4 \times 1 \mathrm{k}$-point grid and found that the difference in total energy is less than $1 \times 10^{-4} \mathrm{eV} /$ atom. Therefore, the $2 \times$ $2 \times 1$ k-point grid is sufficient for the calculation. Similar computational details are described elsewhere [19].

\section{Results and Discussion}

For necessity of later calculations, the equilibrium lattice of $\mathrm{CeSZ}$ was investigated in total energy versus lattice parameter. A stoichiometric $\mathrm{CeZrO}_{4}$ cubic structure in the $1 \times 1 \times 1$ unit cell is employed for the calculation. Figure 1 shows that the total energy of CeSZ bulk was calculated using GGA + $U$ and GGA and plotted as a function of the lattice constant. The effective Hubbard parameter of $3 \mathrm{eV}$ and $5 \mathrm{eV}$ was used in the calculation as the GGA $+U$ approach. The $U=0 \mathrm{eV}$ calculation corresponds to unmodified GGA. It allows a direct comparison of the two methods. Introducing $U$ into the GGA methods results in an increase of lattice constant so that eventually GGA $+U$ may predict the experimental lattice constant $a_{0}=5.34 \AA$ [20]. Therefore, the value of $U_{\text {eff }}=5 \mathrm{eV}$

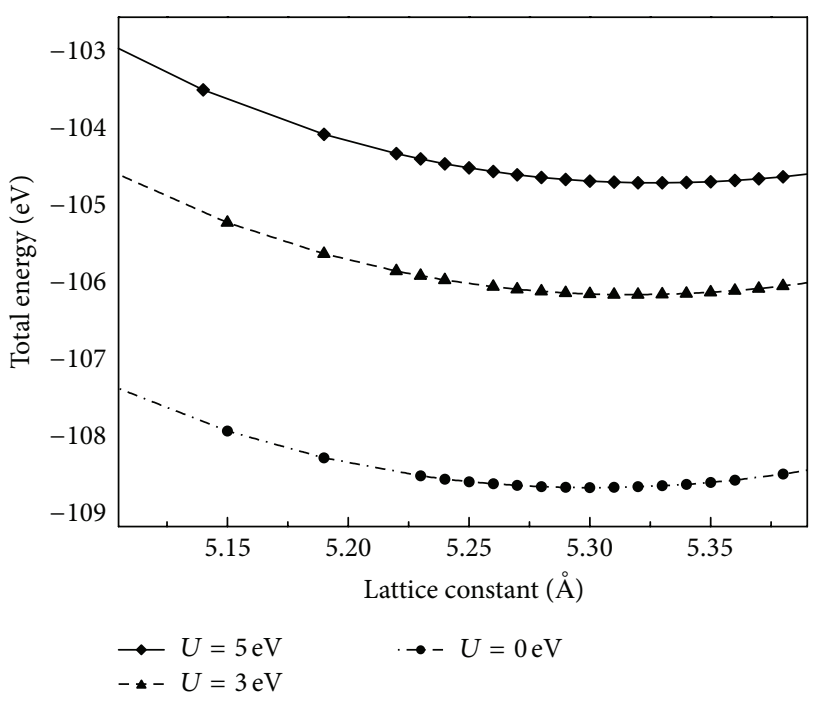

FIGURE 1: The equilibrium lattice constant of CeSZ calculated using the GGA and GGA $+U$.

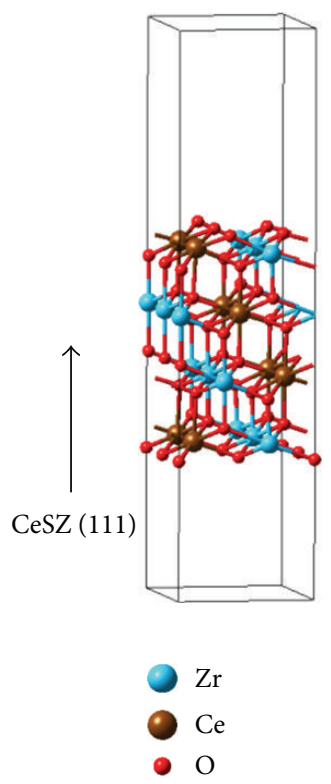

(a)
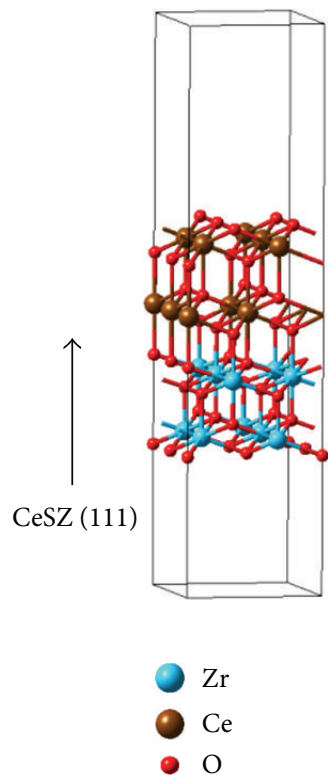

(b)
Figure 2: Schematic illustration of the (a) Ce-segregation-free and (b) Ce-segregated CeSZ (111) surface used for calculations.

as suggested by Nolan et al. [16] was employed for the later calculations.

Based on the calculated lattice constant, the CeSZ (111) surface is constructed using a three-dimensional periodic surface slab mode for the investigation of adsorption phenomena. Both Ce-segregation-free and Ce-segregated surfaces are considered for the theoretical modeling. The optimized structure of the CeSZ (111) surface is shown in Figure 2. The segregation behavior of CeSZ (111) surface has been found in both theoretical and experimental studies [12]. Although some experimental studies such as X-ray photoelectron spectroscopy (XPS) and low energy ion spectroscopy 

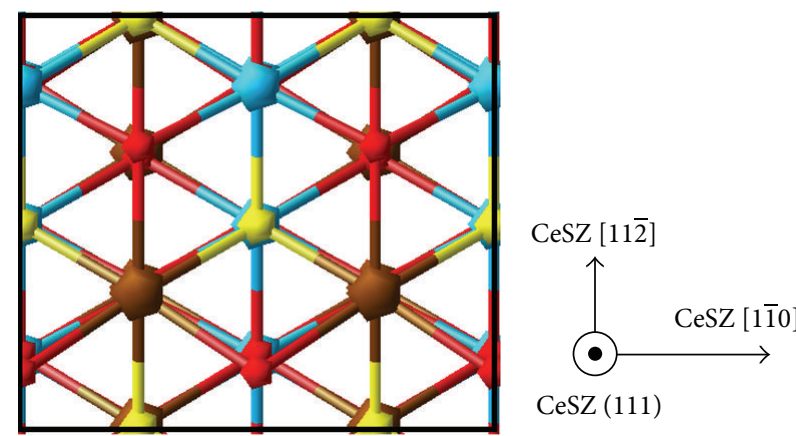

CeSZ (111)

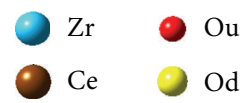

(a)

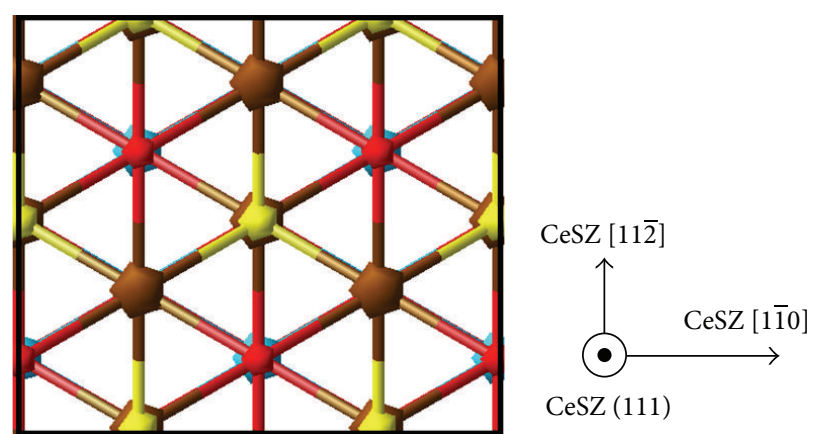

$\begin{array}{ll}\mathrm{Zr} & \bigcirc \mathrm{Ou} \\ \mathrm{Ce} & \mathrm{Od}\end{array}$

(b)

FIGURE 3: Schematic illustration of the adsorption sites for (a) Ce-segregation-free and (b) Ce-segregated CeSZ (111) surfaces.

TABLE 1: Surface energy (in eV/A ${ }^{2}$ ) of Ce-segregation-free and Cesegregated CeSZ (111) surfaces.

\begin{tabular}{lc}
\hline & Surface energy \\
\hline Ce-segregation-free & 0.056 \\
Ce-segregated & 0.044 \\
\hline
\end{tabular}

(LEIS) measurements have found the dopant segregation in zirconia surface $[21,22]$, the atomic structure of the dopant stabilized zirconia surface is still unclear due to the limit of the experimental techniques at present stage. Therefore, the structure of Ce-segregation surface is assumed in this work. All the $\mathrm{Zr}$ atoms in the upper half of the zirconia are replaced with $\mathrm{Ce}$ atoms. Similar assumption has been performed in the case of the Y-segregated YSZ surface structure [19].

The stability of the surface is dependent on the surface energy. The surface energy of the Ce-segregation-free and Cesegregated surfaces was calculated by

$$
E_{\text {surface }}=\frac{\left[E_{\text {slab }}-\left(N_{\text {slab }} / N_{\text {bulk }}\right) E_{\text {bulk }}\right]}{2 A},
$$

where $E_{\text {slab }}$ is the energy of the surface slab, $E_{\text {bulk }}$ is the energy of the bulk unit cell, $N_{\text {slab }}$ is the number of atoms in the slab, $N_{\text {bulk }}$ is the number of atoms in the bulk unit cell, and $A$ is the unit area of the surface. According to (1), a positive and low value of $E_{\text {surface }}$ corresponds to a stable surface. The calculated surface energies of both Ce-segregation-free and Ce-segregated CeSZ (111) surfaces are summarized in Table 1. Surface energy calculations revealed that the structure with the Ce atoms segregation is more energetically stable than that without $\mathrm{Ce}$ atoms segregation. Because the covalent character of $\mathrm{Zr}-\mathrm{O}$ bond is stronger than that of $\mathrm{Ce}-\mathrm{O}$ bond [12], the unsaturated CeSZ (111) surface with breaking of the metal-oxygen bonds should be more easily enriched by $\mathrm{Ce}$ cations than $\mathrm{Zr}$ cations in the top layer.
Calculations of the adsorption energies of In and $\mathrm{N}$ atoms on the two CeSZ (111) surfaces were then performed in order to discuss the growth mechanisms of InN on CeSZ substrates during the initial stage. The adsorption energy $E_{\text {adsorption }}$ is defined as

$$
E_{\text {adsorption }}=E_{\text {substrate }}+E_{\text {adatom }}-E_{\text {total }}
$$

where $E_{\text {total }}$ is the energy of the substrate with an adatom adsorbed onto it, $E_{\text {substrate }}$ is the energy of the substrate without the adatom, and $E_{\text {adatom }}$ is the energy of an isolated adatom. According to (2), $E_{\text {adsorption }}$ generally has a positive value, and a larger value corresponds to stable adsorption. Systematic probing of all symmetric sites on the surfaces is essential for the determination of the adsorption energy. For the Ce-segregation-free surface, nine highly symmetric adsorption sites on the surface were selected for calculations, as shown in Figure 3(a). For the Ce-segregated surface, seven highly symmetric adsorption sites on the surface were selected for calculations, as shown in Figure 3(b). These adsorption sites included the on-top sites (on top of the $\mathrm{Ce}, \mathrm{Zr}$, surface oxygen $\mathrm{Ou}$, and subsurface oxygen $\mathrm{Od}$ ), the bridge sites (in the middle between the $\mathrm{Ce}-\mathrm{Ou}, \mathrm{Zr}$ $\mathrm{Ou}, \mathrm{Ce}-\mathrm{Od}, \mathrm{Zr}-\mathrm{Od}$, and Ou-Od pairs), and the hollow sites (in the center of the $\mathrm{Ce}-\mathrm{Ou}-\mathrm{Od}, \mathrm{Zr}-\mathrm{Ou}-\mathrm{Od}$ triangles). In the calculation, the surface atoms were kept frozen at the positions obtained from the clean surface optimization, and only the positions of the adatoms were fully optimized until the Hellmann-Feynman forces were below $0.05 \mathrm{eV} / \AA$. The calculated adsorption energies of the In and $\mathrm{N}$ atoms on the CeSZ (111) surface are summarized in Tables 2 and 3.

According to the results of adsorption energies, the most stable site for adsorption is a bridge position. It can be seen that the difference in the adsorption energies of In atoms on various sites is less than the difference in the adsorption energies of $\mathrm{N}$ atoms on various sites. We assumed that the barrier to diffusion can be obtained by comparing adsorption energies at different high symmetry sites. This result indicates 


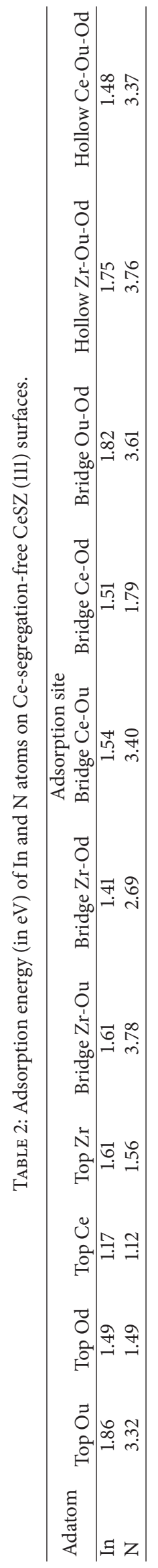


TABLE 3: Adsorption energy (in eV) of In and N atoms on Ce-segregated CeSZ (111) surfaces.

\begin{tabular}{lccccccc}
\hline \multirow{2}{*}{ Adatom } & & & \multicolumn{3}{c}{ Adsorption site } \\
& Top Ou & Top Od & Top Ce & Bridge Ce-Ou & Bridge Ce-Od & Bridge Ou-Od & Hollow Ce-Ou-Od \\
\hline In & 1.74 & 1.40 & 1.26 & 1.47 & 1.41 & 1.70 & 1.55 \\
$\mathrm{~N}$ & 3.48 & 1.19 & 1.07 & 3.58 & 1.98 & 4.10 & 3.37 \\
\hline
\end{tabular}
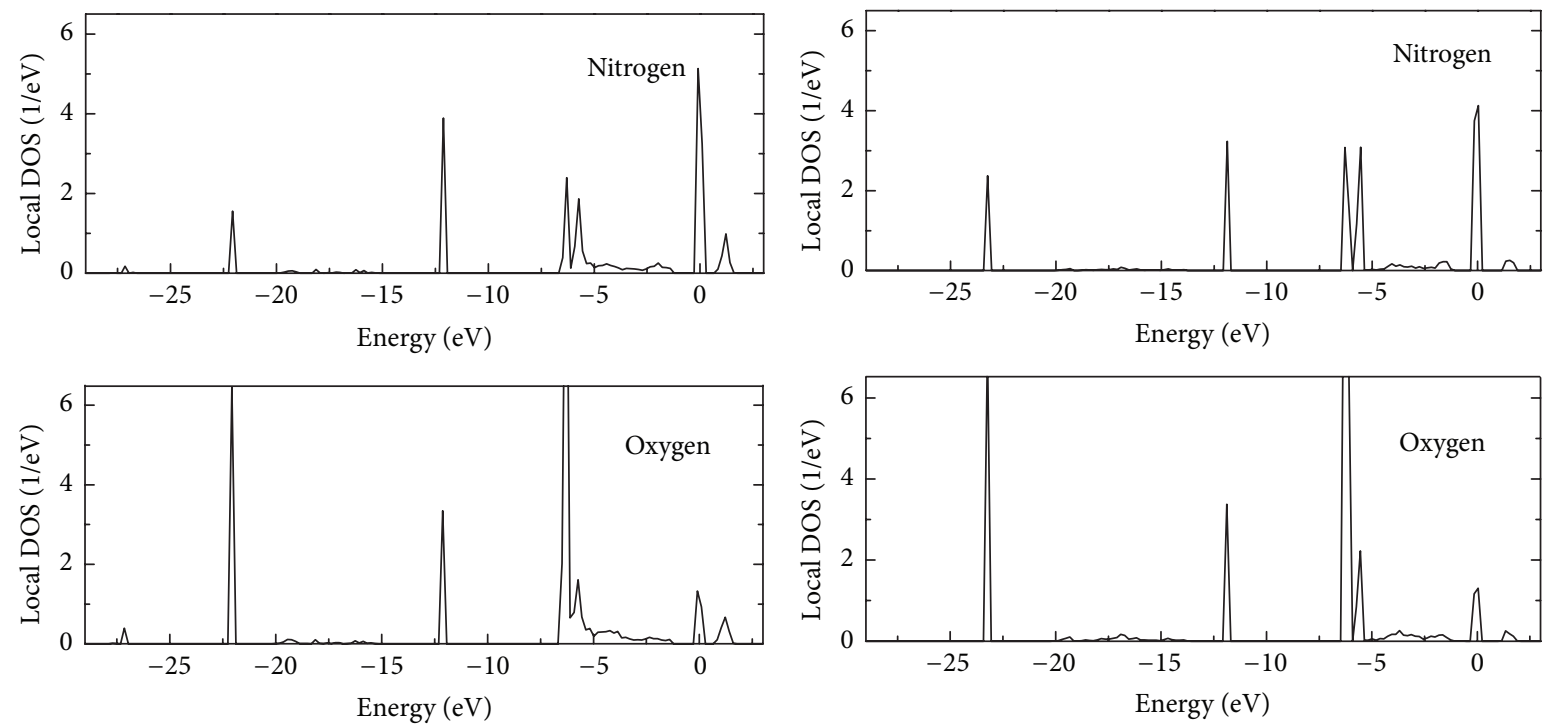

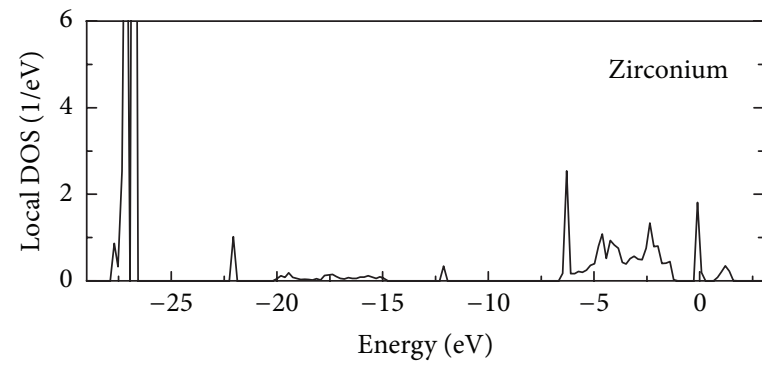

(a)

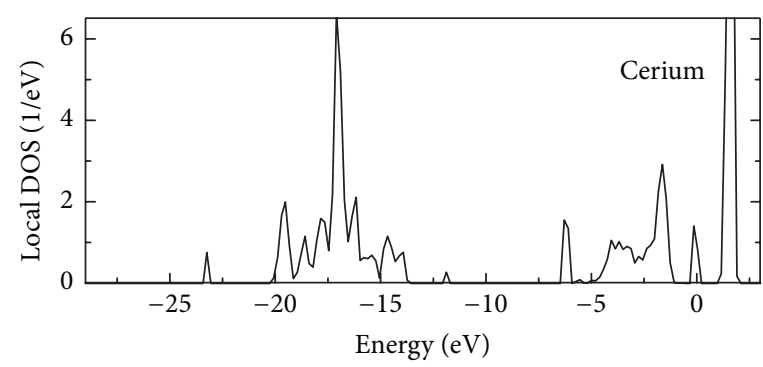

(b)

FIgure 4: The DOS for the structure of a nitrogen atom on (a) Ce-segregation-free and (b) Ce-segregated CeSZ (111) surfaces.

that migration of indium atoms on the surface readily occurs. In contrast, the larger difference in the adsorption energies of $\mathrm{N}$ atoms on various sites suggests that $\mathrm{N}$ atoms tend to stay at the most stable site. These results indicate that the first layer of InN (0001) should be an N layer, and that the in-plane epitaxial relationship is $\operatorname{InN}[11 \overline{2} 0] / / \mathrm{CeSZ}[1 \overline{1} 0]$, which gives a small lattice mismatch between InN (0001) and CeSZ (111), as expected.

Although the higher stability of a nitrogen atom compared to that of an indium atom adsorbed on both Cesegregation-free and Ce-segregated CeSZ (111) surfaces is a common phenomenon, the adsorption energies of the nitrogen atoms are quite different for the different substrates. Thus, to shed light on the interaction mechanism between $\mathrm{N}$ atom and CeSZ (111) surface, the density of state (DOS) for nitrogen adsorption at the most favorable site (Bridge $\mathrm{Zr}$ $\mathrm{Ou}$ ) on the segregation-free surface has been performed, and the results are shown in Figure 4(a). In addition, similar DOS for nitrogen adsorption at the most favorable site (Bridge Ou$\mathrm{Od}$ ) on Ce-segregated surface are shown in Figure 4(b). The atomic DOS of the $\mathrm{N}$ and its nearest $\mathrm{O}$ and cation $(\mathrm{Zr}$ or $\mathrm{Ce}$ ) atoms in the surface region are plotted, respectively. It can be seen that the peaks in the range from -5 to $0 \mathrm{eV}$ belong to $\mathrm{N} 2 p$ orbital; the narrow peak around $-12 \mathrm{eV}$ is assigned to the N2s orbital. In all cases there exists strong hybridization among the $\mathrm{N} 2 p, \mathrm{O} 2 p$, and cation ( $\mathrm{Zr}$ or $\mathrm{Ce}$ ) $d$ orbitals within the energy range from -5 to $0 \mathrm{eV}$. This implies that the strong hybridization is the main mechanism of $\mathrm{N}$ atom interaction with CeSZ (111) surface and determines the value of the adsorption energy for $\mathrm{N}$ atom. The observation is in agreement with our previous research for YSZ (111) surface [19].

Furthermore, for a better characterization of the InN/ CeSZ interfacial structure, we performed the calculation of the adsorption energies of an indium atom on the first nitrogen layer covered CeSZ (111) surfaces to compare the stability of In-polar InN with that of N-polar InN on Ce-segregation-free 
TABLE 4: Adsorption energies (in $\mathrm{eV}$ ) of an In atom on the nitrogen layer covering CeSZ (111) surfaces.

\begin{tabular}{lcc}
\hline Surface & \multicolumn{2}{c}{ Adsorption energy of In atom } \\
In-polarity & N-polarity \\
\hline Ce-segregated & 3.32 & 3.26 \\
Ce-segregation-free & 3.95 & 3.66 \\
\hline
\end{tabular}

and Ce-segregated surfaces, respectively. In fact, the lattice polarity of the $\mathrm{InN}$ is determined by the adsorption site for $\mathrm{In}$ atom. The In-polarity $\mathrm{InN}$ is modeled by the adsorption of an indium atom at the center of three nitrogen atoms (Hollow $\mathrm{N}$ site). By comparison, In atom adsorption on the top of a nitrogen atom (Top $\mathrm{N}$ site) corresponds to $\mathrm{N}$-polarity $\mathrm{InN}$ [19].

The adsorption energies of an In atom at the "Hollow N" and "Top N" sites on both Ce-segregation-free and Ce-segregated CeSZ (111) surfaces are summarized in Table 4. It is found that the adsorption energy for In-polar InN is larger than that for N-polar InN on both two CeSZ surfaces. The growth of In-polar InN is energetically preferred. Moreover, the difference between In- and N-polar InN for Ce-segregated surface is smaller than that for Ce-segregation-free surface. It indicates that the polarity conversion between Inand $\mathrm{N}$-polar InN on Ce-segregated substrate during growth easily occurred due to the low energy barrier. The difference in polarity determination on Ce-segregation-free and Ce-segregated surfaces is generally attributed to the Cesegregation on the surface. The different $d$-orbital electrons of $\mathrm{Zr}$ and Ce will affect the adsorption of a nitrogen atom on CeSZ surface. Moreover, the adsorption of an indium atom on the first nitrogen layer covered CeSZ surface. The lattice polarity determination is dependent on the surface segregated Ce atoms.

\section{Conclusion}

In this study, we have investigated the growth mechanism of InN on a CeSZ (111) surface by first-principles calculations. N atoms have larger adsorption energies than In atoms on both Ce-segregation-free and Ce-segregated CeSZ (111) surface. The results imply that the first layer at the initial stage of growth should be a nitrogen layer. The theoretical studies have revealed that the epitaxial relationships of $\operatorname{InN}(0001)$

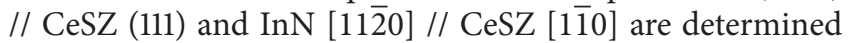
by the adsorption process of nitrogen atoms on the surfaces at the initial stage of the growth. The DOS analysis reveals that there is a clear strong hybridization among the $\mathrm{N} 2 p$, $\mathrm{O} 2 p$, and cation ( $\mathrm{Zr}$ or Ce) $d$ orbitals. It is responsible for the main mechanism of $\mathrm{N}$ atom interaction with CeSZ (111) surface and determines the interface structure. Moreover, the mechanism of the determination of InN polarity on CeSZ (111) substrates was studied by theoretical calculations. It was found that the growth of In-polar InN is energetically more stable than that of $\mathrm{N}$-polar InN. The difference in polarity determination is determined by different $d$-orbital electrons of $\mathrm{Zr}$ and $\mathrm{Ce}$ atoms.

\section{Competing Interests}

The authors declare that there is no competing interests regarding the publication of this paper.

\section{Acknowledgments}

This work was supported by the National Natural Science Foundation of China (Grant no. U1404217), the Foundation of He'nan Educational Committee (Grant no. 16B430001), and the Program of Department of Science and Technology of He'nan Province (Grant no. 152102210195).

\section{References}

[1] S. L. Wang, H. X. Liu, X. Song, Y. L. Guo, and Z. N. Yang, "An analytical model of anisotropic low-field electron mobility in wurtzite indium nitride," Applied Physics A: Materials Science and Processing, vol. 114, no. 4, pp. 1113-1117, 2014.

[2] K. Okubo, A. Kobayashi, J. Ohta, M. Oshima, and H. Fujioka, "Electron mobility of ultrathin InN on yttria-stabilized zirconia with two-dimensionally grown initial layers," Applied Physics Letters, vol. 102, no. 2, Article ID 022103, 2013.

[3] X. Wang, S. Liu, N. Ma et al., "High-electron-mobility InN layers grown by boundary-temperature-controlled epitaxy," Applied Physics Express, vol. 5, no. 1, Article ID 015502, 2012.

[4] W. A. Hadi, P. K. Guram, M. S. Shur, and S. K. O’Leary, “Steadystate and transient electron transport within wurtzite and zincblende indium nitride," Journal of Applied Physics, vol. 113, no. 11, Article ID 113709, 2013.

[5] J. Kioseoglou, E. Kalesaki, G. P. Dimitrakopulos, T. Kehagias, P. Komninou, and T. Karakostas, "Atomistic modeling and HRTEM analysis of misfit dislocations in InN/GaN heterostructures," Applied Surface Science, vol. 260, pp. 23-28, 2012.

[6] N. Nepal, N. A. Mahadik, L. O. Nyakiti et al., "Epitaxial growth of cubic and hexagonal InN thin films via plasma-assisted atomic layer epitaxy," Crystal Growth and Design, vol. 13, no. 4, pp. 1485-1490, 2013.

[7] P. Wang, Y. Yuan, C. Zhao et al., "Lattice-polarity-driven epitaxy of hexagonal semiconductor nanowires," Nano Letters, vol. 16, no. 2, pp. 1328-1334, 2016.

[8] T. Honke, H. Fujioka, J. Ohta, and M. Oshima, "InN epitaxial growths on Yttria stabilized zirconia (111) step substrates," Journal of Vacuum Science \& Technology A, vol. 22, no. 6, pp. 2487-2489, 2004.

[9] M. Oseki, K. Okubo, A. Kobayashi, J. Ohta, and H. Fujioka, "Field-effect transistors based on cubic indium nitride," Scientific Reports, vol. 4, article 3951, 2014.

[10] M. O. Zacate, L. Minervini, D. J. Bradfield, R. W. Grimes, and K. E. Sickafus, "Defect cluster formation in $\mathrm{M}_{2} \mathrm{O}_{3}$-doped cubic $\mathrm{ZrO}_{2}$," Solid State Ionics, vol. 128, no. 1-4, pp. 243-254, 2000.

[11] D. R. Sellick, A. Aranda, T. García et al., "Influence of the preparation method on the activity of ceria zirconia mixed oxides for naphthalene total oxidation," Applied Catalysis B: Environmental, vol. 132-133, pp. 98-106, 2013.

[12] R. G. Crespo, N. H. De Leeuw, S. Hamad, and U. V. Waghmare, "Phase separation and surface segregation in ceria-zirconia solid solutions," Proceedings of the Royal Society A: Mathematical, Physical and Engineering Sciences, vol. 467, no. 2131, pp. 1925-1938, 2011. 
[13] X. Wei, C. Dong, Z. Chen, K. Xiao, and X. Li, "Density functional theory study of $\mathrm{SO}_{2}$-adsorbed $\mathrm{Ni}(111)$ and hydroxylated $\mathrm{NiO}\left(\begin{array}{lll}1 & 1 & 1\end{array}\right)$ surface," Applied Surface Science, vol. 355, pp. 429435, 2015.

[14] J. P. Perdew, K. Burke, and M. Ernzerhof, "Generalized gradient approximation made simple," Physical Review Letters, vol. 77, no. 18, pp. 3865-3868, 1997.

[15] P. E. Blöchl, "Projector augmented-wave method," Physical Review B, vol. 50, no. 24, pp. 17953-17979, 1994.

[16] M. Nolan, "Modifying ceria (111) with a $\mathrm{TiO}_{2}$ nanocluster for enhanced reactivity," The Journal of Chemical Physics, vol. 139, no. 18, Article ID 184710, 2013.

[17] A. Christensen and E. A. Carter, "First-principles study of the surfaces of zirconia," Physical Review B, vol. 58, no. 12, pp. 80508064, 1998.

[18] H. J. Monkhorst and J. D. Pack, "Special points for Brillouinzone integrations," Physical Review B, vol. 13, no. 12, pp. 51885192, 1976.

[19] Y. Guo, S. Inoue, A. Kobayashi, J. Ohta, and H. Fujioka, "Theoretical investigation of the polarity determination for cplane InN grown on yttria-stabilized zirconia (111) substrates with yttrium surface segregation," Applied Physics Express, vol. 6, no. 2, Article ID 021002, 2013.

[20] M. Kuhn, S. R. Bishop, J. L. M. Rupp, and H. L. Tuller, "Structural characterization and oxygen nonstoichiometry of ceriazirconia $\left(\mathrm{Ce}_{1-\mathrm{x}} \mathrm{Zr}_{\mathrm{x}} \mathrm{O}_{2-\delta}\right)$ solid solutions," Acta Materialia, vol. 61, no. 11, pp. 4277-4288, 2013.

[21] A. Bernasik, K. Kowalski, and A. Sadowski, "Surface segregation in yttria-stabilized zirconia by means of angle resolved X-ray photoelectron spectroscopy," Journal of Physics and Chemistry of Solids, vol. 63, no. 2, pp. 233-239, 2002.

[22] M. de Ridder, R. G. van Welzenis, A. W. Denier van der Gon et al., "Subsurface segregation of yttria in yttria stabilized zirconia," Journal of Applied Physics, vol. 92, no. 6, pp. 3056-3064, 2002. 

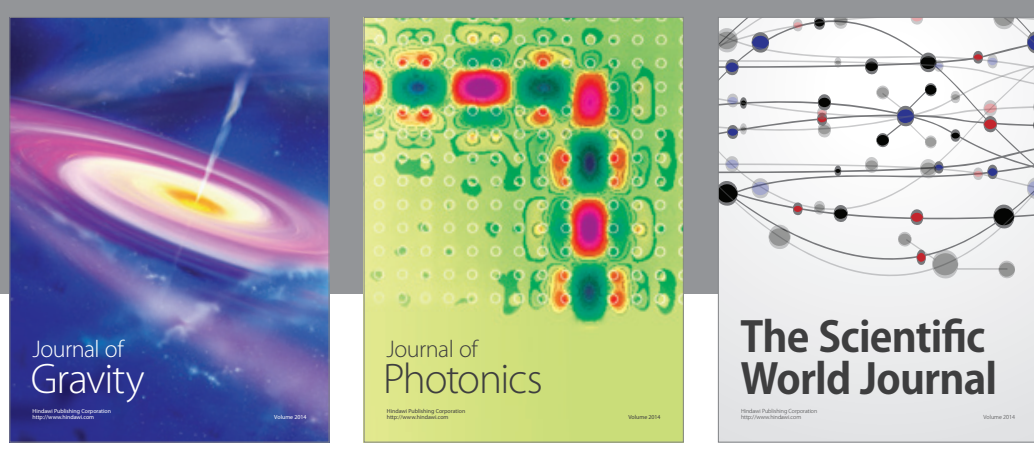

The Scientific World Journal
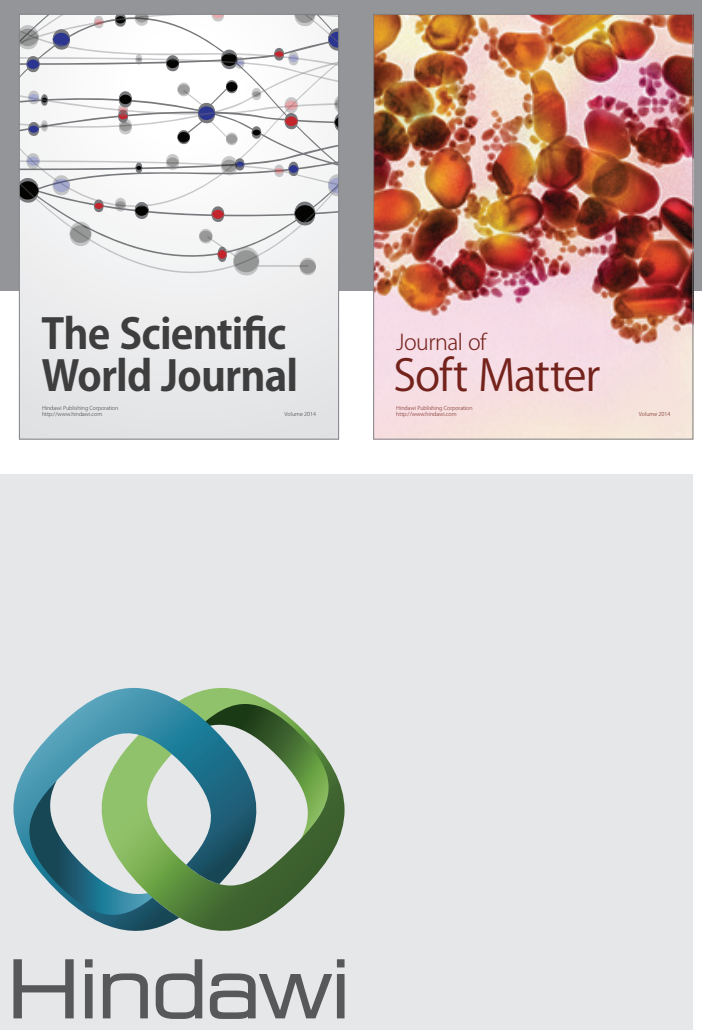

Submit your manuscripts at

http://www.hindawi.com

nternational Journal of

Statistical Mechanics
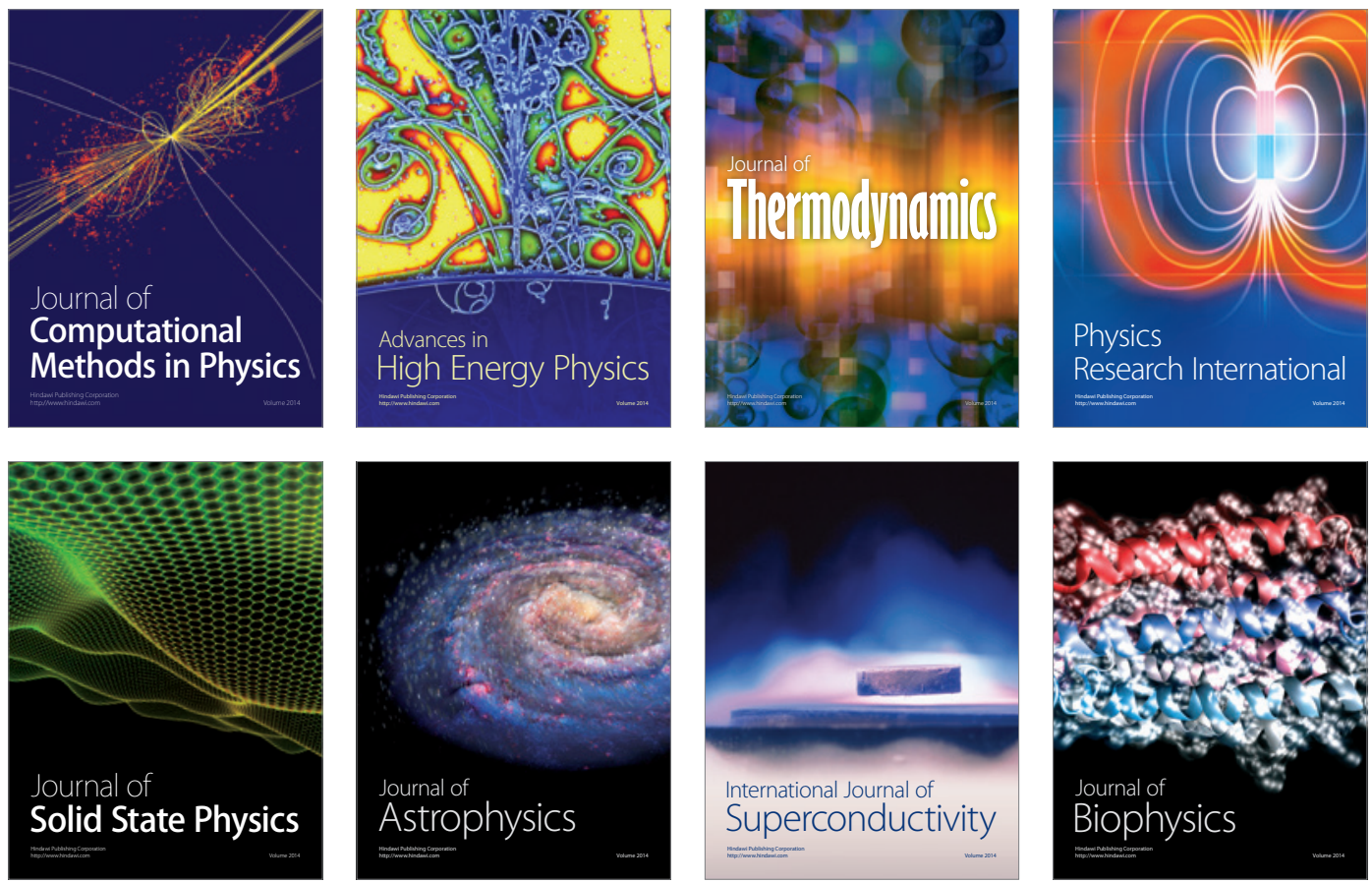
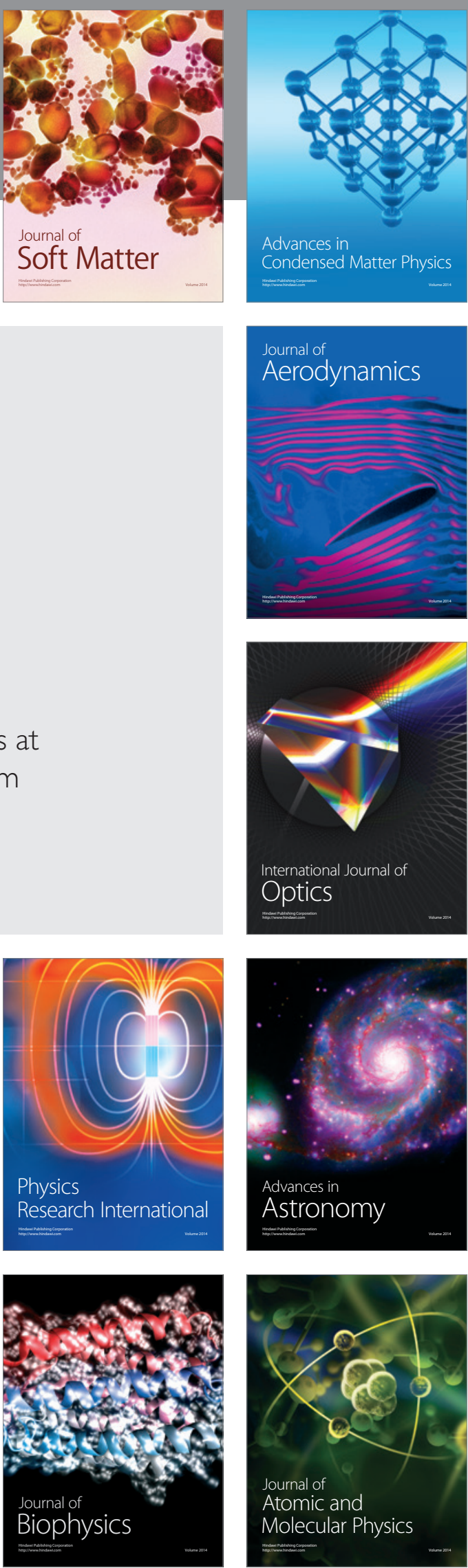